\section{HONOURS, AWARDS, APPOINTMENTS}

Ruby Austin Shield

Nehaba Rathod has been awarded the London Deanery's Ruby Austin Shield for the best case presentation of 2012 in her DF1 year. Nehaba, who is on the Charing Cross Dental Foundation training scheme, presented a case to raise awareness of the oral implications of Khat chewing, focusing on the most recent evidence base behind clinical decisions such as her decision to use a natural tooth pontic following the extraction of an anterior tooth.

\section{Post-CCST fellowship}

Dr Ahmed Al-Khayatt has been awarded the first postCCST fellowship in restorative dentistry by the NorthWest Deanery. Dr Al-Khayatt will take up the post to gain additional experience in head and neck cancer rehabilitation following completion of his Certificate of Completion of Specialist Training (CCST) at the London Deanery.

\section{Young Dental Writer of the Year}

The British Dental Editors Forum (BDEF) has announced that the winner of this year's BDEF/British Dental Trade Association (BDTA) Young Dental Writer of the Year Award is Ms Laura Hatton. Laura was nominated for her article The truth from the trenches which was published in the Dental Tribune in 2011. Second place was awarded to dentist Mr Alexander Holden who was nominated for the article Lost in transition - changes in communication in the leap from dental student to foundation dentist which was published in the BDJ (2011; 211: 459-461).

\section{DENTAL NURSE TAKES STAR TURN}

Marianna Dadejova, special care dental nurse with the Special Care and Community Dental services, North East London NHS Foundation Trust (NELFT), was one of 10,000 talented volunteers taking part in the Opening Ceremony of the London 2012 Olympic Games.

Marianna, 29, played a nurse in the sequence representing Britain's National Health Service.

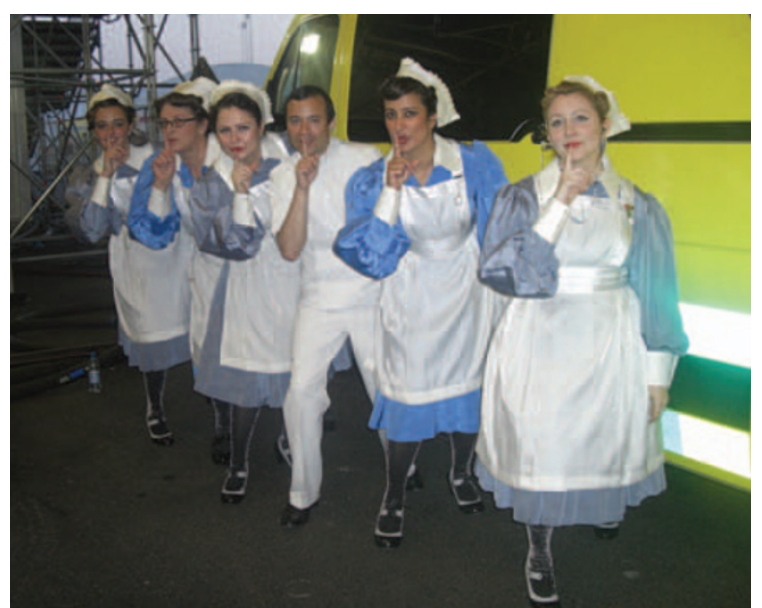

Marianna Dadejova, special care dental nurse (third from left), with fellow performers at the 2012 Olympic Games Opening Ceremony

\title{
EASTMAN STUDIES OLYMPIANS' ORAL HEALTH
}

A research team led by UCL Eastman Dental Institute has been investigating the oral health of elite athletes at the London 2012 Olympic Games and the impact of oral health on training and performance.

To date, more than 300 athletes have been recruited to take part, making it one of the most comprehensive investigations of oral health in elite athletes ever undertaken.

Professor Ian Needleman, leading the study, explained: 'There are many potential threats to oral health in athletes including exercise-induced immunosuppression, difficulty in taking time away from training for oral care, and drinks high in sugars. Despite this, oral health does not usually appear on the radar for many athletes and little is known about such impacts on their performance.

'Several athletes during the Olympic Games [at the time of reporting] have told us how big an effect it has had on them and we will look forward to analysing the data over the next few months.'

Professor Needleman is working with Dr Paul Ashley, Dr Aviva Petrie, Professor Stephen Porter and Professor Nikolaos Donos, and the London 2012 Polyclinic dental team led by Professor Farida Fortune from Barts and The London School of Medicine and Dentistry.

The research continues the UCL Eastman Dental Institute's focus on investigating the relationship between oral health and general health and well-being.

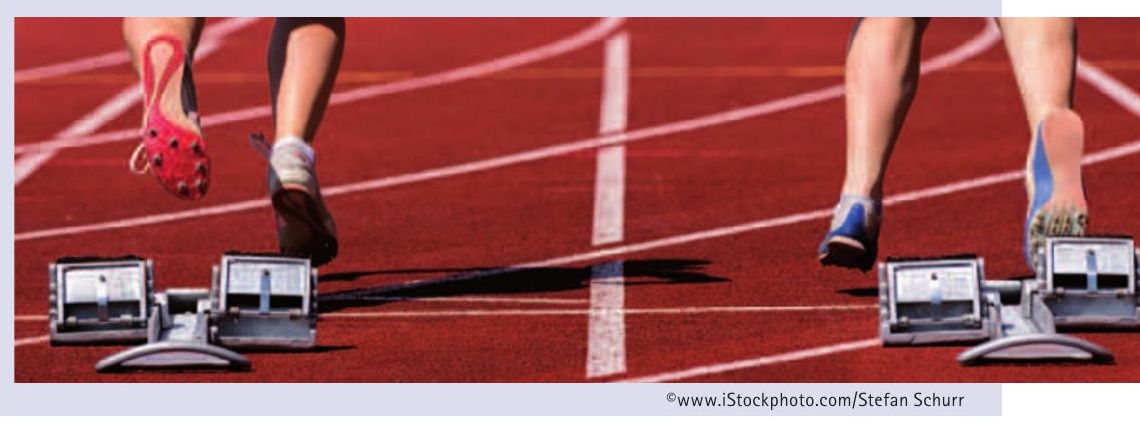

\section{PRESIDENT TURNS PILGRIM FOR ORTHODONTICS}

The President of the British Orthodontic Society (BOS), Keith Pearson, is raising money for research which will be targeted at finding the evidence base for the value and importance of orthodontic treatment to patients, both young and old.

In June Dr Pearson, who is an Orthodontic Specialist in practice in Beckenham, walked as a 'pilgrim' for nine days along The Way of St James to Santiago de Compostela in north-western Spain, covering $180 \mathrm{~km}$ and arriving in time for the European Orthodontic Meeting.

In communication with BOS during his walk, one of $\mathrm{Dr}$
Pearson's updates reported that he had spent 'overnight in a very small village built almost entirely of granite and considered to be one of the wettest and windiest in Spain! Tuesday was slightly less arduous in that the weather has improved but still overcast and cold but only drizzle today. Overnight in a rectory just outside Sarria...'

The orthodontic research will directly benefit all those in clinical practice in the years to come as pressures mount on the funding of the provision of such treatment, particularly within the NHS.

To donate please visit www.justgiving.com/KeithPearsonsWalk. 